\title{
Oscillatory network coding of a global stimulus.
}

\author{
Brent Doiron ${ }^{a, b}$, André Longtin ${ }^{a}$, and Benjamin Lindner ${ }^{a}$ \\ ${ }^{a}$ Physics, U. Ottawa,150 Louis Pasteur, Ottawa, Canada, K1N 6N5 \\ ${ }^{b}$ Cell. and Molec. Med., U. Ottawa, 451 Symth Rd., Ottawa, Canada, K1H 8M5
}

\begin{abstract}
The pyramidal cells of weakly electric fish respond to environmental broadband electrical stimuli. They have recently been shown to exhibit oscillations in mean firing rate in response to global stimuli that affect the whole body simultaneously similar to communication stimuli for these animals. In contrast, for spatially localized stimuli such as those produced by prey, the firing rate simply fluctuates around a constant mean. ${ }^{?}$ This switch in coding strategy relies on delayed negative (inhibitory) feedback connections in the neural network. We first summarize these experimental findings, as well as our mathematical modeling of this effect using a globallycoupled delayed inhibitory network of leaky integrate-and-fire neurons (LIF's). Here we study the mechanism of the transition from oscillatory to non-oscillatory firing states in such networks. This is done using simulations of a simpler network of LIF's with current based Gaussian white noise stimuli, rather than conductance based bandlimited Gaussian stimuli. We focus on the effect of feedback gain, current bias, and stimulus intensity on the oscillation under global conditions, and see how the decrease of these parameters brings on a response characteristic of the local case. These simulations are performed for a fixed amount of individual synaptic noise to each cell. We also show how insights into these results can be obtained from the analysis of stimulus-induced oscillations in a simpler rate model description of this spatially-extended excitable system.
\end{abstract}

Keywords: Nonlinear dynamics, noise, neuron, inhibition, weakly electric fish, oscillation, integrate-and-fire model, feedback, coding, Hopf bifurcation

\section{INTRODUCTION}

In order to survive in complex environments organisms must discriminate between sensory inputs with varying spatiotemporal structure. Weakly electric fish offer a simple case study of this phenomenon, since prey stimuli are spatially localized (hereafter refered to as local stimuli) whereas communication stimuli are spatially diffuse (hereafter refered to as global stimuli). In the time-domain, the stimuli of interest are often considered as bandlimited Gaussian noises for simplicity? ${ }^{?}$ It has recently been shown? ${ }^{?}$ that the sensory circuitry of one species of weakly electric fish, Apteronotus Leptorhynchus, can produce two distinct kinds of output firing patterns to stimuli with Gaussian temporal structure, depending on the local/global categorisation of the spatial aspect of the stimulus. Local stimuli produce a noisy Poisson-like firing pattern with some mean firing rate and yields a unimodal inter-spike interval (ISI) probability density. Global stimuli, on the other hand, produce an oscillatory firing pattern, in which the firing can still be considered noisy, yet the rate clearly oscillates around a mean value. Futhermore the ISI probability density are distinctly bimodal suggestive of a stochastic burst discharge. Thus, the neural circuitry of periphery sensory stages can differentially encode global versus local stimuli, and accordingly, communication versus predation, using the presence/absence of spike train oscillations. This study focusses on a simplified network model that oscillates in reponse to a spatially global stimuli with Gaussian spectral characteristics. In contrast to our more detailed model ${ }^{?}$, here we assume that the global stimulus is Gaussian white noise, rather than bandlimited Gaussian noise. Further, we assume that the feedback enters the current balance equation in an additive manner, rather than multiplicative; while this latter form is a better representation of synaptic input, its multiplicative nature makes the theoretical analysis of the network dynamics more difficult. Since such an analysis is one of our eventual goals, the present study serves to pinpoint

Further author information: (Send correspondence to B.D.)

B.D.: E-mail: bdoiron@physics.uottawa.ca, Telephone: 1613 562-5800 x8258

A.L.: E-mail: alongtin@physics.uottawa.ca, Telephone: 16135625800 x6762 
the features of the detailed model in? which are key for the observed phenomena. In section II we provide some background on our recent modelling of the oscillatory coding. Next we introduce a simpler leaky integrate-and-fire network model, and show how inhibitory feedback gain, intrinsic cell firing rate, and global stimulus intensity can control the oscillatory behavior of this system in response to a global stimulus. Section III introduces a further simplified rate description of the network, which allows for mean field theory to be derived. It is shown that the deterministic mean field undergoes a supercritical Hopf bifurcation as both driving input and feedback gain are increased. A stochastic forcing of the mean field then shows how a noise-induced oscillation occurs as a global stimulus intensity grows. Finally section IV gives some concluding remarks.

\section{BACKGROUND}

In ${ }^{?}$ we show the specific cells that exhibit the aforementioned switch in coding are the pyramidal cells of a structure known as the electrosensory lateral line lobe (ELL). These cells receives input from the outside world via an array of electroreceptors in the skin of the fish. ELL pyramidal cells also project to higher brain areas, but also, importantly, receive feedback input from these areas (for a review see?). This well-characterized circuitry underlies our recent neural network model which predicted that simple, diffuse, delayed and fastacting inhibitory feedback could support the presence of oscillations for global input. This prediction was experimentaly verified when reversible blockade of feedback inhbition removed oscillatory behavior in response to communication stimuli.? By "diffuse delayed feedback" we mean the following. The output of all ELL cells drives cells in another neuron population; these in turn inhibit indiscrimately all ELL cells after a time delay representing long range axonal connections and synaptic delays. Our original study used bandlimited Gaussian white noise input to mimick the temporal characteristics of the physical (electrical) environmental stimuli. Here we consider a simplified version of such a network in which the stimulus is now Gaussian white noise. Consider a nonautonomous network of $N$ leaky integrate-and-fire (LIF) neurons coupled via diffuse delayed pulsatile inhibition. Let the membrane potential of the $i^{t h}$ neuron at time $t$ is given by $V_{i}(t)$. The dynamics of $V_{i}(t)$ evolve according to the following stochastic delay differential equation:

$$
\frac{d}{d t} V_{i}(t)=-V_{i}(t)+\mu_{i}+\sqrt{2 D_{i}} \xi_{i}(t)+\sigma_{s} \xi_{s}(t)-\frac{g}{N} \sum_{j} \sum_{m} \alpha^{2}\left(t-t_{j m}-\tau_{d}\right) e^{\alpha\left(t-t_{j m}-\tau_{d}\right)} \Theta\left(t-t_{j m}-\tau_{d}\right)
$$

Eq (??) gives a standard LIF neuron with time measured in units of membrane time constant. A "spike" occurs when the voltage $V_{i}$ reaches an imposed threshold $V_{t h}$. The spike is recorded as a time $t_{i m}$ where $m$ is the spike number for cell $i$. We have nondimensionalized $V_{i}$ to be in units of $V_{t h}$ so that $V_{t h}=1$. After a spike occurs $V_{i}$ is reset to 0 and remains at 0 for a period $\tau_{r}$ modeling an absolute refractory period. The excitabilty of each cell is determined by a bias current term $\mu_{i}$; in this study we assume $\mu_{i}=\mu$ for all cells. Each cell has its internal source of noise, considered here as a zero-mean Gaussian white noise for simplicity (they were OrnsteinUhlenbeck processes in our early study ${ }^{\text {? }}$ ), with standard deviation $\sigma_{i}=\sqrt{2 D_{i}}$, and $D_{i}$ is assumed to be the same for all cells. The cells also receive the environmental stimulus which is also considered as a zero-mean Gaussian white noise with standard deviation $\sigma_{s}=\sqrt{2 D_{s}}$. In our paper, we study how the oscillations depend on certain parameters of the system, all the while applying the stimulus noise to all cells, as in the global configuration. Some of the resulting firing patterns will mimick those seen under local conditions, even though the input is global throughout our study (i.e. spatially homogeneous). The last term in the model, i.e. the feedback term, is a sum of alpha functions, each of which is activated $\tau_{d}$ time units (the feedback delay) after a neuron in the network has fired. $\Theta$ is the standard Heaviside function where $\Theta(t)=0$ for $t<0$ and $\Theta(t)=1$ for $t>0$; this imposes a causality between LIF spike discharge and synaptic response. The gain of the feedback pathway is set by the parameter $g$. Note that this feedback input is the same for all cells, mimicking the all-to-all, or diffuse, nature of the connectivity. We will use two statistics to assess the presence of oscillations in our network. The interspike interval probability density (PD) is a first order statistic. From our previous study, we know that it makes a transition from unimodel for the non-oscillatory case to bimodal in the oscillatory case. The power spectral density (PSD) of the sum of the spike trains of all neurons, which is a second order statistic, will also be

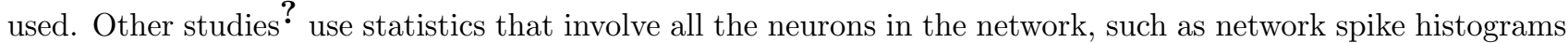
for example. We have used this measure in our previous work ${ }^{?}$ however in the present study we probe network 
activity with statistics from one representative cell in the network. Such an approach is better suited to motivate future experimental studies since multi-unit recordings are difficult.

\section{RESULTS WITH LIF MODEL}

In Fig.1, we show the effect of feedback gain $g$ on the network oscillation. Without feedback (open loop), the neurons lack any influence over one another and thus the spike time statistics as shown by the PSD and PD are those of a LIF neuron driven by Gaussian white noise of standard deviation $\sqrt{2 D_{i}}+\sigma_{s}$. In particular, the PSD shows no oscillatory behavior in the spike train and the PD is unimodal in character. In contrast, when $g=100$ (closed loop) we have a distinct oscillation in the spike train at approximately $25 \mathrm{~Hz}$ and a well-defined bimodality in the PD. The timescale of the oscillation is naturally sensitive to the exact value of the feedback

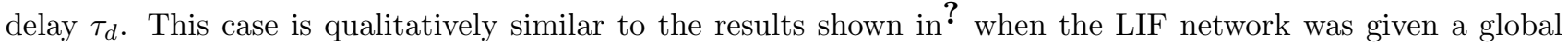
stimulus. We note that the mean firing rate of the network increases in the absence of inhibition. This can be seen by observing the power in the spike train at high frequencies $(>80 \mathrm{~Hz})$ and noting that in the limit of large frequency the PSD is equal to the mean firing rate of the neuron. ${ }^{?}$ In Fig.2, we show the effect of
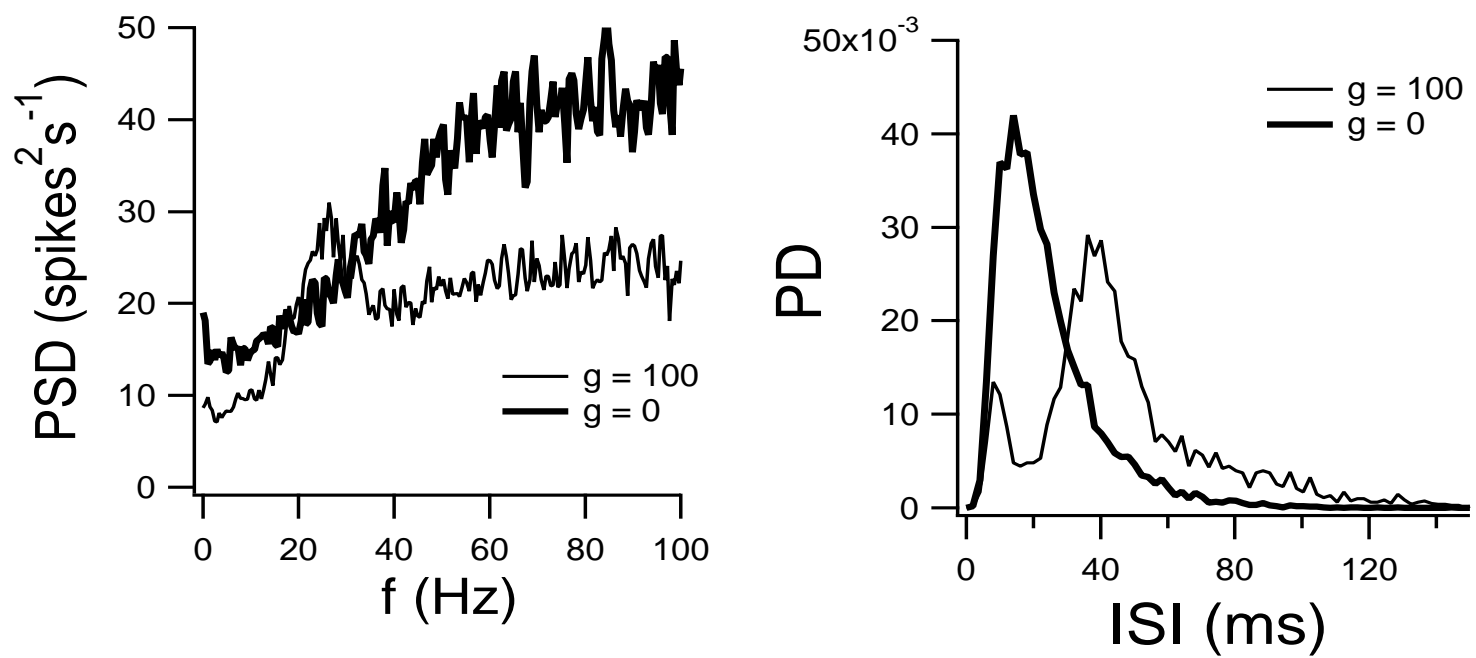

Figure 1. Effect of feedback gain. Spike train power spectral density PSD (left) and ISI probability distribution PD (right) when inhibitory gain is $g=100$ (closed loop) and $g=0$ (open loop). Other model parameters $N=100, \mu=0.9$, $\alpha=3, \tau_{d}=1, \tau_{r}=0.1, D_{i}=0.03, \sigma_{s}=0.12$. We have assumed a membrane time constant of $10 \mathrm{~ms}$. All network simulations were computed using an Euler-Maruyama scheme with a fixed time step of $\Delta t=0.005$.

the intrinsic bias current $\mu$ on the network oscillation. As $\mu$ is reduced from 0.9 to 0.7 the firing rate of the neuron reduces by about a factor of 2 and interestingly the oscillatory characteristic of the spike train observed from the PSD and the bimodality of the PD are significantly compromised. Upon further reduction of $\mu$ it is possible to enhance this effect so that the oscillation disappears completely (results not shown). Finally, in Fig. 3 we show the effect of the stimulus intensity $\sigma_{s}$ on the network oscillation. When $\sigma_{s}$ is set to 0 (autonomous network) the spike train no longer oscillates and the ISI structure is unimodal. The firing rate of the network also decreases as expected from the significant change in membrane fluctuation when $\sigma_{s}=0$. Taken together, these results show that the potential of the network to respond in an oscillatory manner to a global stimulus is dependent upon (at least) network connectivity, intrinsic membrane excitabilty, and stimulus characteristics. The fine structure of these dependencies can be further explored using simulations of our complex stochastic network model. However, we have found a simple dependence of the oscillation strength on three parameters, and it is of interest to understand this behavior in an analytically simpler framework that avoids the spiking nonlinearities of LIF neurons. In particular, the results of Figs. 1-3 motivate a simple rate model ${ }^{\text {of }}$ of the network dynamics where some of these issues can be addressed. This is the focus of the next section. 

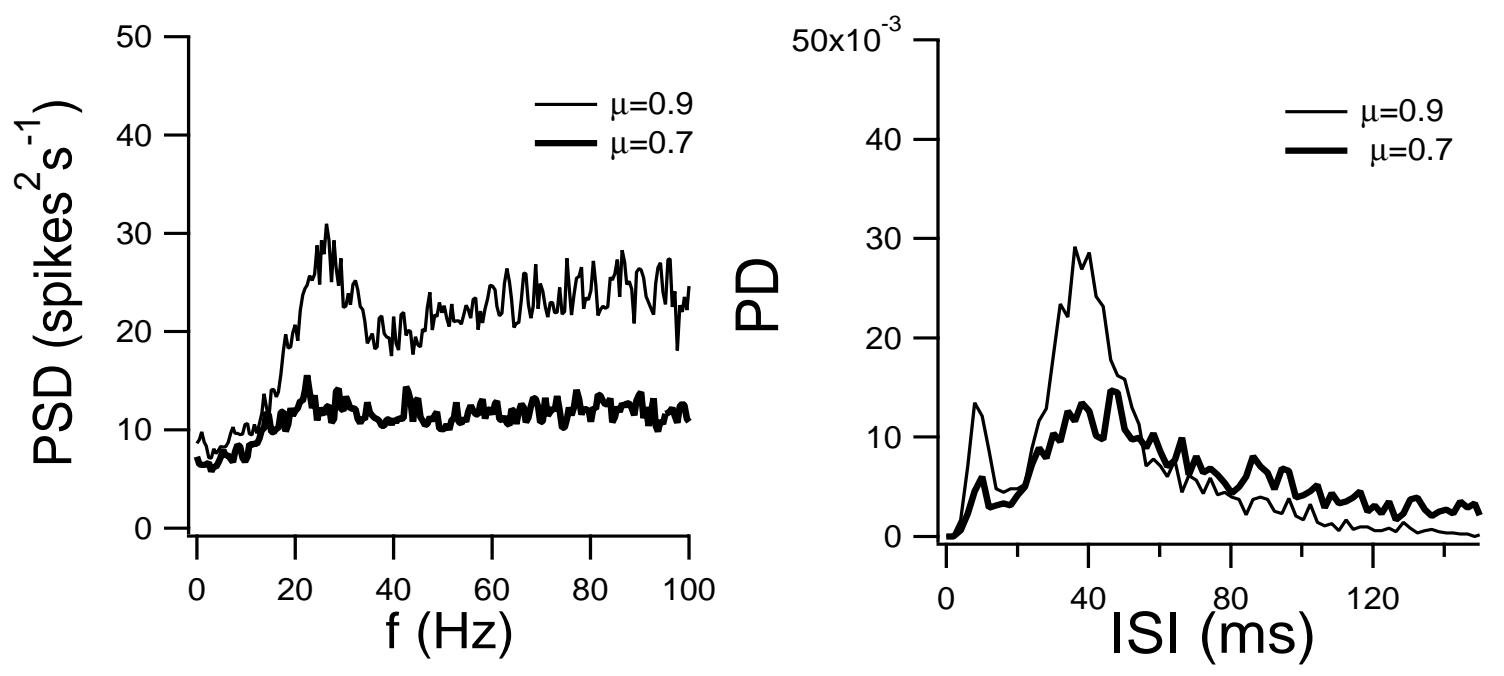

Figure 2. Effect of bias current. Spike train power spectra $P S D$ (left) and ISI probability distribution PD (right) when is $\mu=0.9$ and $\mu=0.7 . g=100$ and other parameters are as in figure 1 .
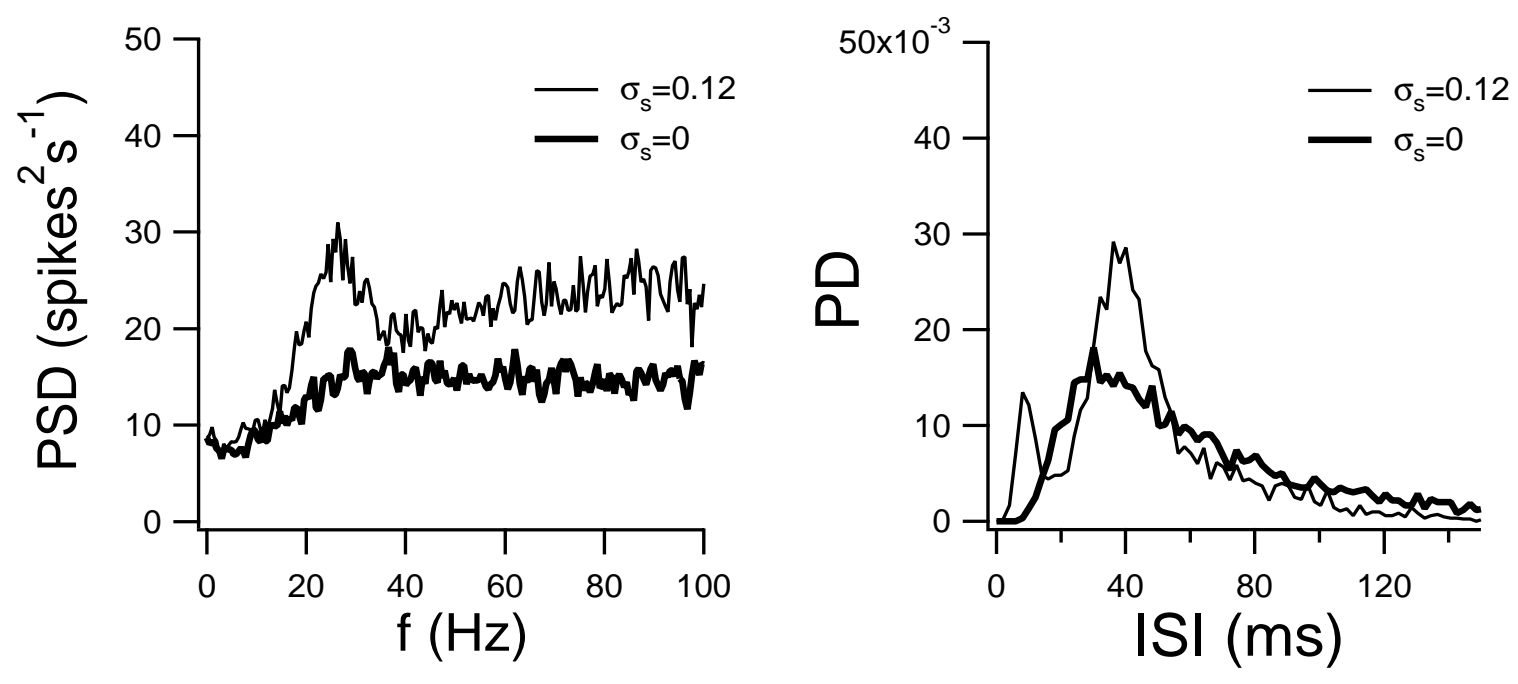

Figure 3. Effect of stimulus intensity. Spike train power spectra density PSD (left) and ISI probability distribution PD (right) when is $\sigma_{s}=0.12$ and $\sigma_{s}=0 . g=100$ and other parameters are as in figure 1.

\section{RATE MODEL}

We now consider a network of $N$ neurons where the dynamics of each neuron is described by a rate model. This differs from an LIF description in that the representative state variable of each neuron is firing rate rather than membrane potential. Similar to the LIF network we coupled all cells via a delayed "mean rate" which mimicks a global inhibitory feedback projecting to all cells. Specifically the dynamics of the firing rate of the $i^{t h}$ neuron at time $t$, labelled $r_{i}(t)$, is governed by

$$
\frac{d r_{i}(t)}{d t}=-\frac{r_{i}(t)}{\tau_{m}}+\mu+\sqrt{2 D_{i}} \xi_{i}(t)+\sigma_{s} \xi_{s}(t)-g G\left(R\left(t-\tau_{d}\right)\right)
$$




$$
R(t)=\frac{1}{N} \sum_{i=1}^{N} r_{i}(t)
$$

where $R(t)$ is the mean field, or average firing rate, of the network at time $t$. The effective "membrane" time constant is given by $\tau_{m}$ as in the LIF model and $\mu_{i}$ is a time independent input to cell $i$. $\mu_{i}$ represents the steady state, or spontaneous, firing rate of cell $i$ in the absence of feedback or time dependent forcing. $\xi_{i}(t)$ and $\xi_{s}(t)$ are stochastic processes with means $\left\langle\xi_{i}(t)\right\rangle=\left\langle\xi_{i}(t)\right\rangle=0$ and with correlations $\left\langle\xi_{i}(t) \xi_{j}\left(t^{\prime}\right)\right\rangle=\delta_{i j} \delta\left(t-t^{\prime}\right)$, $\left\langle\xi_{s}(t) \xi_{s}\left(t^{\prime}\right)\right\rangle=\delta\left(t-t^{\prime}\right)$, and $\left\langle\xi_{i}(t) \xi_{s}\left(t^{\prime}\right)\right\rangle=0 . \xi_{i}(t)$ represents an intrinsic Gaussian white noise source specific to each cell with intensity given by $D_{i}$. In contrast $\xi_{s}(t)$ is Gaussian white noise of variance $\sigma_{s}$ shared by all cells $; \xi_{s}(t)$ may be viewed as a stochastic global stimulus. Finally $G$ is the network coupling function of which the argument is the network mean field $R$ evaluated at a delay $\tau_{d}$ in the past. To motivate the coupling function $G$ we consider the network of cells projecting their outputs uniformly to a distant region of the brain. This region then integrates the ouput and projects back uniformly to the network with a delay $\tau_{d}$ modelling the finite velocity of axonal transmission and delays due to chemical transimission at a synapse. In this intepretation the function $G$ is the input/output relation (or f-I curve) of this distant region and, for the purposes of this study, we consider the projection as strictly inhibitory with strength $g>0$ (the inhibition is due to the minus sign in Eq ??). As is common in such network studies we choose $G$ to be

$$
G(R(t))=\frac{1}{2}[\tanh (\alpha R(t)+\beta)+1]
$$

Multiplying Eq (??) by $1 / N$ and summing over all the elements and taking the limit $N \rightarrow \infty$ gives the dynamics of the mean field in the thermodynamic limit as:

$$
\frac{d R(t)}{d t}=-\frac{R(t)}{\tau_{m}}+\sigma_{s} \xi_{s}(t)+\mu-\frac{g}{2}\left[\tanh \left(\alpha R\left(t-\tau_{d}\right)+\beta\right)+1\right]
$$

Note that the influence of the intrinsic noise sources $\xi_{i}(t)$ on the mean field dynamics is negligible when $N$ is large, however the common stimulus $\xi_{s}(t)$ remains. We will study network oscillations by understanding first the deterministic $\left(\sigma_{s}=0\right)$ behavior of Eq (??) and then following with the full stochastic case $\left(\sigma_{s}>0\right)$.

\subsection{Deterministic network}

Let $\sigma_{s}=0$ so that the network evolves deterministically. We nondimensionalize the system Eq (??) with the transformation $R \rightarrow \alpha(R-\beta)$ and $t \rightarrow \frac{t}{\tau_{d}}$. This gives

$$
\frac{d R(t)}{d t}=-\kappa R(t)-\kappa \bar{g} \tanh (R(t-1))+\kappa(\bar{\mu}-\bar{g})
$$

with

$$
\bar{\mu} \equiv \alpha\left(\tau_{m} \mu-\beta\right) ; \bar{g} \equiv \frac{g \tau_{m} \alpha}{2} ; \kappa \equiv \frac{\tau_{d}}{\tau_{m}}
$$

We drop the ${ }^{-}$to simplify notation. We will analyze Eq (??) using standard nonlinear dynamics techniques as appropriate for scalar delay differential systems. ${ }^{?}$ Fixed points, $R^{*}$, of the system are given by the roots of the following

$$
-R^{*}+(\mu-g)-g \tanh \left(R^{*}\right)=0
$$

This equation only admits one real root for all values of $g>0$ and $\mu$. To indicate the dependence of this point on $\mu$ and $g$, we relabel the fixed point as $R_{\mu, g}^{*}$. Linearizing about the fixed point gives the following local dynamics

$$
\frac{d R(t)}{d t}=-\kappa R(t)-h R(t-1)
$$

where $\left.h \equiv g \frac{d}{d R} \tanh (R)\right|_{R=R_{\mu, g}^{*}}=g\left(1-\tanh ^{2}\left(R_{\mu, g}^{*}\right)\right)$. Note that $g \geq h \geq 0$ for all $g>0$ and $\mu$. The anastz $R(t)=A e^{\lambda t}$ (with $A$ some constant) gives the following characteristic equation for Eq (??)

$$
\lambda+\kappa\left(1+\mu e^{-\lambda}\right)=0
$$


In general, $\lambda$ is complex; let $\lambda=a+i b$. Substitution of this into Eq (??) gives

$$
\begin{aligned}
a & =-\kappa\left(1+h e^{-a} \cos b\right) \\
b & =\kappa h e^{-a} \sin b
\end{aligned}
$$

Consider the case when $a=0$, from Eqs (??) and (??) we have

$$
\begin{aligned}
1+h \cos b & =0 \\
b & =\kappa \sqrt{h^{2}-1}
\end{aligned}
$$

For $b$ in $R$ we require that $|h| \geq 1$ and since we have shown above that $h \geq 0$ we have that the case of interest is $h \geq 1$. From this restriction it can be shown that Eqs (??) and (??) can be satisfied an infinite number of times by the following sequence

$$
\begin{aligned}
b_{k} & =\arccos \frac{1}{-h_{k}}+2 \pi k \\
\mu_{k} & =\sqrt{\left(\frac{b_{k}}{\kappa}\right)^{2}+1}
\end{aligned}
$$

with $k$ in $N_{0}$. For eack $k$ we have a pair of conjugate eigenvalues, $\lambda_{k}= \pm i b_{k}$, that lie on the imaginary axis. Differentiating Eqs (??) and (??), rearranging terms, and setting $a=0$ gives

$$
\left(\left.\frac{\partial a}{\partial \mu}\right|_{a=0}\right)_{k}=\frac{\kappa\left(\cos b_{k}-\tau h_{k}\right)}{2 \kappa h_{k} \cos b_{k}-\left(\kappa^{2} h_{k}^{2}+1\right)}
$$

The Hopf bifurcation theorem for delayed systems is similar to the case for non-delayed systems. ${ }^{?}$ In order for a system to be at a Hopf bifurcation we require that (1) $a=0,(2) b_{k} \neq 0$, and (3) $\left(\left.\frac{\delta a}{\delta h}\right|_{a=0}\right)_{k} \neq 0$. Condition (1) is satisfied by assumption and condition (2) by substituting that $h \geq 1$ into Eq (??). It is simple to show that condition (3) is satisfied for any $k$ in $R$. Consider the converse of condition (3), i.e that $\left(\left.\frac{\delta a}{\delta h}\right|_{a=0}\right)_{k}=0$. Then we have from Eq (??) the following relation

$$
\cos b_{k}-\kappa h_{k}=0
$$

Substituting $b_{k}$ from Eq (??) and rearraging terms in Eq (??) gives that $h_{k}^{2}=-\frac{1}{\kappa}$. This cannot be satisfied for $h \geq 1$ and $\kappa>0$, thus $\left(\left.\frac{\delta a}{\delta h}\right|_{a=0}\right)_{k} \neq 0$ for all $k$ in $N$. Eq (??) shows a countably infinite number of Hopf bifurcations, one for each $h=h_{k}$. However, we are only interested in the case when $R_{\mu, g}$ loses stability. This occurs when $\operatorname{Re}(\lambda)<0$ for all $\lambda$ satisfying Eq (??) and then two conjugate $\lambda$ s pass across the imaginary axis as $h$ goes through $h_{k}$ for some $k$ in $N$. We contend that this occurs for $k=0$ in the series given in Eqs (??) and (??). Letting $k=0$ and fixing $\kappa$, substituting $b_{0}$ from Eq (??) into Eq (??), and rearranging gives $h_{0}$ as the root of the following trancendental equation

$$
h_{0}^{2}-1-\left(\frac{\arccos \frac{-1}{h_{0}}}{\kappa}\right)^{2}=0
$$

Eq (??) has one real root for all $h_{0}$. In Fig. 4 we plot a curve Hopf bifurcations in $g$ - $\mu$ parameter space. This is accomplished by first determining $h_{0}$ for a given $\kappa$ using standard root finding methods on Eq (??). We then again use root finding methods to solve Eq (??) for the $\mu$ and $g$ values which give $\left|h-h_{0}\right|$ to be within some small tolerence $\left(10^{-4}\right)$. This curve partitions parameter space into oscillatory (upper part) and non-oscillatory (lower part) regimes. The "U"-like shape of the curve suggests that for a fixed $g$ it is possible to switch from stable to oscillatory behavior as $\mu$ is varied near 0 and to return to stable behavior as $\mu$ grows large. This is demonstated in the Fig. 5 where we show the bifurcation diagram of Eq (??) for $g=5$. Two distinct supercritical Hopf bifurcations are shown, one for low $\mu$ and the other for high $\mu$. The interpretation of the second bifurcation is that the driving force is so strong that the inhibitory feedback fails to influence the mean field dynamics. This is caused by the eventual saturation of inhibition at high firing rates imposed by our choice of $G$ (Eq ??). Note how such behavior does not occur as we increase $g$; only one bifurcation is possible. For the next section we will focus on the first Hopf bifurcation which occurs only for $\mu$ near 0 . 


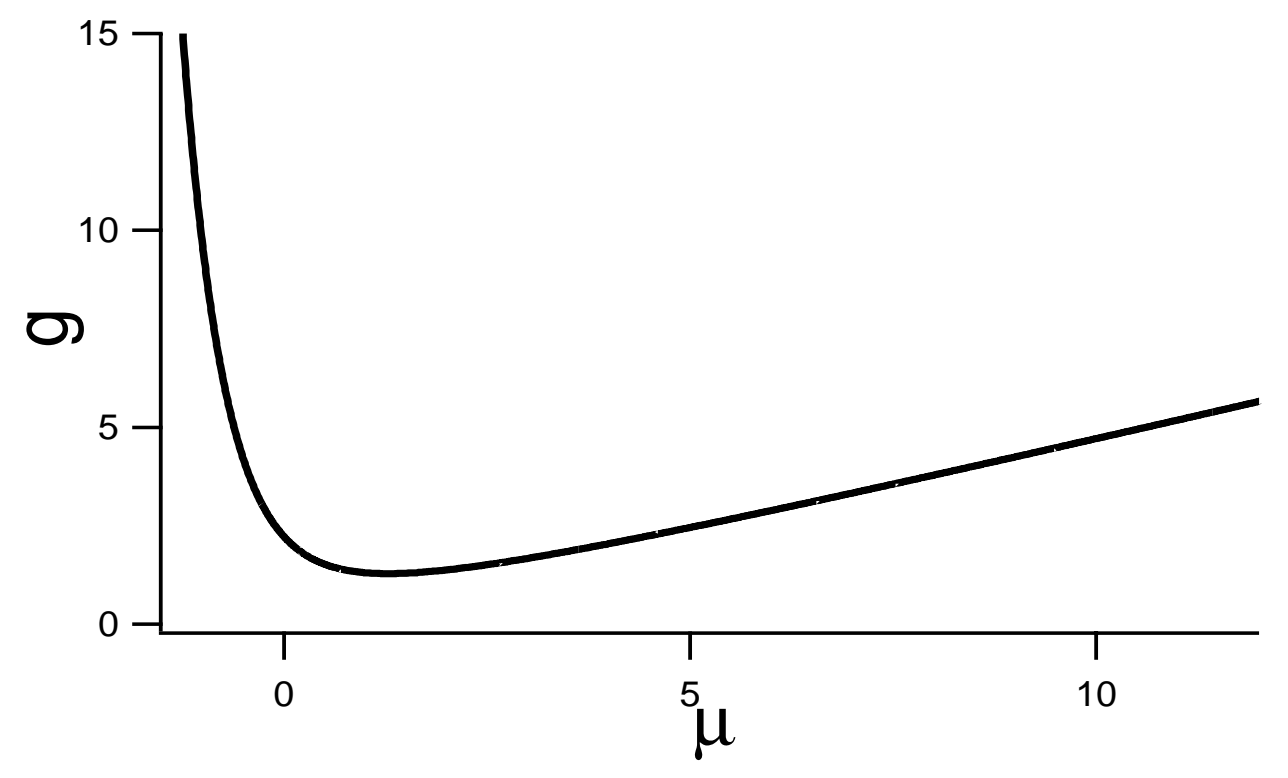

Figure 4. Boundary in the gain-bias subspace of parameter space for the rate model. Crossing this boundary from below produces a Hopf bifurcation from a fixed point (constant firing rate) to a limit cycle (oscillatory firing rate). The relative delay $\kappa$ was set to 3 .

\subsection{Stochastic Stimuli and noise induced oscillations}

We now consider the case of $\sigma_{s}>0$ so that the network evolves with a stochastic driving force. Using the same set of transformations as the previous section we rewrite Eq (??) as

$$
\frac{d R(t)}{d t}=-\kappa R(t)-\kappa g \tanh (R(t-1))+\kappa\left(\mu-g+\bar{\sigma}_{s} \xi_{s}(t)\right)
$$

where $R, \mu$, and $g$ are as in the previous section and $\overline{\sigma_{s}} \equiv \alpha \tau_{m} \sigma_{s}$. Again we drop the ${ }^{-}$notation for simplicity. In Fig. 6 we show the stochastic dynamics of $R(t)$ when the system is subthreshold but near to the Hopf bifurcation $(\mu=-0.8, g=5)$. A noise induced oscillation is observed for $\sigma_{s}>0$, such behavior for delay systems is well

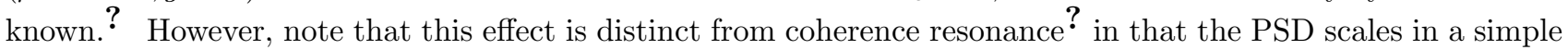
multiplicative manner with $\sigma_{s}$ for moderate values of $\sigma_{s}$. This is standard behavior for linear stochastic delay differential equations. At present the effect of the nonlinearity in $G$ upon the stochastic dynamics of Eq (??) remains unclear. Further work will be presented in subsequent publications.

\section{CONCLUSION}

We have presented an analysis of oscillatory behavior in a network of LIF neurons with global delayed feedback; in contrast to our previous study, the input to this network was a spatially homogeneous additive Gaussian white noise. The oscillation is found to increase in strength as the feedback gain, the bias current, or as the stimulus intensity increases. Similar findings are also explained by a simpler mean field rate model of neural firing in this network. Such a simplified network provides insight into the induction of oscillations by the stimulus noise in the vicinity of a Hopf bifurcation in such an extended globally-coupled excitable system. We comment here on how the LIF network and rate network differ in two important ways. First, the feedback in the LIF model is linear, i.e summation of independent $\alpha$-functions. This is in contrast to the feedback of the rate network which is nonlinear (Eq (??)). Second, the intrinsic dynamics of the LIF model are quite nonlinear due to the influence of the threshold, whereas the intrinsic dynamics of the rate equations do not have this nonlinearity. How this "transfer" of nonlinearity from the single cell to the network connectivity affects oscillatory discharge 


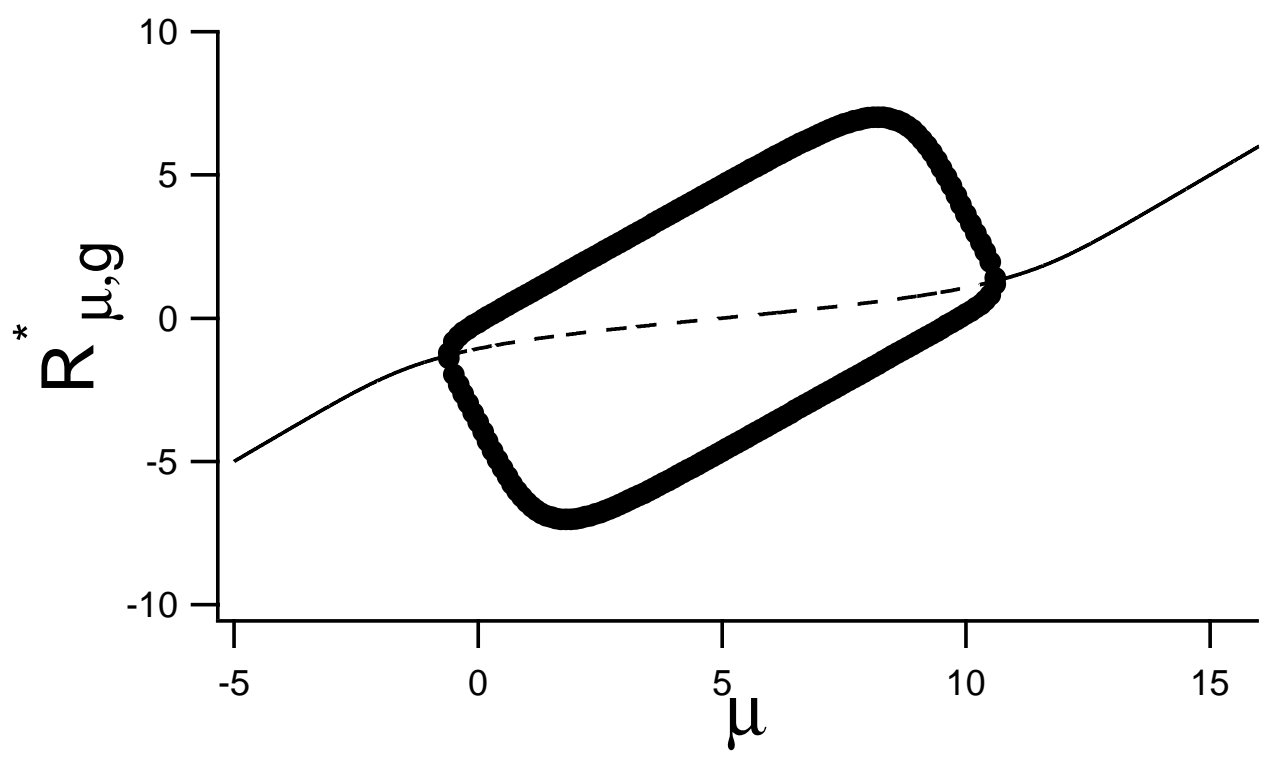

Figure 5. Bifurcation diagram for the rate model. The asymptotic firing rate is plotted as a function of the bias current. A Hopf bifurcation to an oscillatory firing rate occurs as the bias increases, and a reverse Hopf bifurcation occurs for an even higher bias. The dashed line represents the unstable fixed point. The upper and lower branches correspond to the maximal and minimal amplitude of the limit cycle.

is, at the moment, unclear. Nevertheless, certain qualitative features are present in both systems indicating that the analytic simplicity of the rate formalism can offer some insight on the issue of stimulus-induced spike train oscillations to global stimuli. From a neural systems perspective, while changes in parameters $g$, $\mu$, and $\sigma_{s}$ can affect pyramidal cell spike discharge in similar ways, they are representative of quite distinct operations. A shift in $g$ is typical of a plastic change in synaptic strength which can occur over a variety of time scales in these animals. ' However, a change in $\mu$ models shifts in membrane excitability; recently, ELL pyramidal cells have displayed intrinsic mechanisms in which spike (and burst) thresholds can vary significantly within a single cell.? Finally, changes in $\sigma_{s}$ mimic either the effects of a feedforward gain control mechanism? ${ }^{?}$ or more simply the distance between the animal and the stimulus (conceiving of $\xi_{s}(t)$ as mimicking communication $\sigma_{s}$ would decay as communicating fish move away from one another). Thus understanding an abstract model of global stimulus induced oscillation which places all such operations in a simpler framework could produce predictions that span a range of neural behavior.

\section{ACKNOWLEDGMENTS}

The authors acknowledge support from NSERC (B.D.; A.L.) and OPREA (A.L.; B.L.) as well as useful conversations with Maurice Chacron, Len Maler, Joseph Bastian, and Jason Middleton.

\section{REFERENCES}

1. B. Doiron, M.J. Chacron, L. Maler, A. Longtin, and J. Bastian, "Inhibitory feedback required for network oscillatory responses to communication but not prey stimuli" Nature 421, pp. 539-543, 2003.

2. F. Gabbiani, W. Metzner, R. Wessel, and C. Koch, "From stimulus estimation to feature extraction in weakly electric fish" Nature 384, pp. 564-567, 1996.

3. N. Berman, and L. Maler, "Neural architexture of the electrosensory lateral line lobe: adaptation for coincidence detection, a sensory searchlight, and frequency-dependent adaptive filtering" J. Exp. Biol. 202, pp. 1243-1253, 1999. 

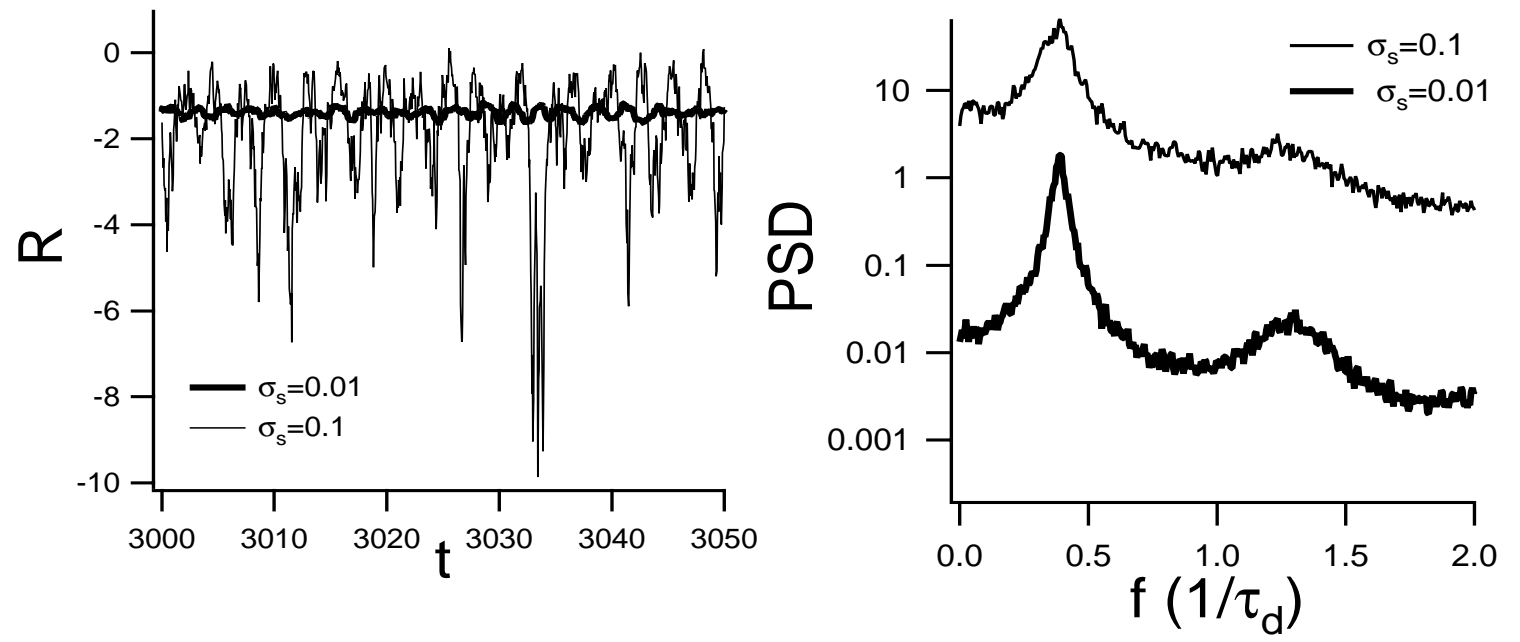

Figure 6. Noise-induced oscillations. Time series of $R(t)$ (left) and associated power spectral density of $\mathrm{R}(\mathrm{t})$ (right) for three different stimulus intensities $\left(\sigma_{s}\right)$. The determinsitic system $\left(\sigma_{s}=0\right)$ corresponding to these simulations is shown and is subthreshold to the Hopf bifurcation at $\mu=-0.8, g=5$. All network simulations were computed using an Euler-Maruyama scheme with a fixed time step of $\Delta t=0.001$. Note that $R(t)$ can be negative due to the transformation used in Eq (??)

4. N. Brunel, "Dynamics of sparsely connected networks of excitatory and inhibitory spiking neurons" J. Comput. Neurosci. 8, pp. 183-208, 2000.

5. J. Franklin, and W. Bair, "The effect of refractory period on the power spectrum of neuronal discharge" SIAM J. Appl. Math. 55, pp. 1074-1101, 1995.

6. P. Dayan, and L.F. Abbott, "Theoretical Neuroscience". MIT Press, Cambridge, Mass., 2001.

7. F. Giannakopoulos and A. Zapp, "Local and global Hopf bifurcation in a scalar delay-differential equation" J. Math. Anal. Appl. 237, pp.425-450 (1999).

8. J.K. Hale and S.V. Lunel, "Introduction to Functional Differential Equations" Springer Verlag, New York, 1993.

9. A. Pikovsky, and J. Kurths, "Coherence resonance in a noise-driven excitable system" Phys. Rev. Lett. 78, pp. 775-779, 1997.

10. A. Longtin, "Noise-induced transitions at a Hopf bifurcation in a first order delay-differential equation" Phys. Rev. A. 44, pp. 4801-4813, 1991.

11. J. Bastian "Plasticity of feedback inputs in the apteronotid electrosensory system" J. Exp. Biol. 202, pp. 1327-1337, 1999.

12. B. Doiron, L. Noonan, N. Lemon, and R.W. Turner, "Persistent Na+ current modifies burst discharge by regulating conditional backpropagation of dendritic spikes" J. Neurophysiol. 89, pp. 324-337, 2003. 